\title{
Kasabach-Merritt syndrome combined with hypercalcemia: A case report
}

\author{
RUNYING ZOU, FANG PENG, TIAN YU, SAIZHEN ZENG, YALAN YOU, KEKE CHEN, \\ HUI ZOU, XIN TIAN, CHENGGUANG ZHU and XIANGLING HE \\ Department of Hematology and Oncology of Children's Medical Center, \\ Hunan Provincial People's Hospital, Changsha, Hunan 410005, P.R. China
}

Received November 4, 2016; Accepted August 4, 2017

DOI: $10.3892 /$ etm.2017.5332

\begin{abstract}
The present case report presented the diagnosis and treatment course of an infant diagnosed with Kasabach-Merritt syndrome (KMS) combined with hypercalcemia (HC). A 35-day-old infant with swelling on the upper right arm for $>1$ month and thrombocytopenia for 1 day was admitted to Hunan Provincial People's Hospital (Changsha, China) and a series of treatments, including $\gamma$-globulin impact, heparin anticoagulation, platelet transfusion, supplement of cryoprecipitate and fibrinogen following heparinization and inhabitation of vascular endothelial cell proliferation by propranolol, were performed. At 2 months after the initial admission to the hospital, surgery was conducted and the hemangioma was removed through pipeline arteriosclerosis embolization when the patient was hospitalized again with symptoms of vomiting and atrophy accompanied by HC. The level of blood calcium reduced to normal following surgery. Cases of KMS combined with HC are extremely rare and the most effective way to treat such cases is surgical resection of the hemangioma.
\end{abstract}

\section{Introduction}

Congenital vascular lumps were previously generally referred to as hemangioma; however, these vascular abnormalities were classified in detail in 1982 by Mulliken and Glowacki in Harvard University (1). Based on different biological characteristics of endothelial cells and histopathological features, as well as clinical manifestations, vascular diseases are divided into two distinct categories: Hemangiomas and vascular

Correspondence to: Professor Xiangling He, Department of Hematology and Oncology of Children's Medical Center, Hunan Provincial People's Hospital, 61 JieFang West Road, Changsha, Hunan 410005, P.R. China

E-mail: hexiangl@163.com

Key words: Kasabach-Merritt syndrome, hemangioma, thrombocytopenia, hypercalcemia malformations, and both may cause Kasabach-Merritt syndrome (KMS) (2-4). KMS was firstly reported in 1940 by Kasabach and Merritt (5), and is a relatively rare disease accounting for only $1 \%$ of all hemangiomas (6). Hemangiomas was responsible for platelet trapping, which, by abnormally proliferating endothelium within the hemangioma, can result in the activation of platelets with secondary activation of coagulation cascades, eventually leading to consumption of various clotting factors (7). An immunohistochemical study using monoclonal antibody against CD61, a marker of platelets, and isotope studies using 111indium-labeled platelets and $51 \mathrm{Cr}$-labeled platelets support the possible role of platelet trapping in the development of KMS (8). Patients with KMS develop local tumor intravascular coagulation, which leads to severe thrombocytopenia, coagulation disorders, anemia and systemic inflammatory responses (9). Clinical manifestations of KMS, including local or systemic subcutaneous and visceral bleeding, high flow heart failure and red or purple vascular tumors, could be combined with laboratory blood tests, imaging or pathological examinations for diagnosis of this disease (10).

The key to ensure effective treatment of KMS is to remove or shrink the vascular tumors. Improving the condition of disseminated intravascular coagulation and thrombocytopenia combined with glucocorticoid therapy remains the first-line treatment course of KMS (11). For patients who are insensitive to glucocorticoid therapy, other therapeutic methods, including interferon therapy, $\beta$-blockers, chemical treatment, anti-platelet drugs, radiotherapy, supportive care or combination therapy of glucocorticoids, could be given as alternative treatments. The International Association for the Study of Vascular Anomalies (ISSVA) classification method for vascular diseases may also be adapted to KMS, and the majority of pathological types of vascular tumors associated with KMS are acquired tufted angioma (ATA) or Kaposi hemangioendothelioma (KHE) (12). KHE, possessing features of hemangioma and Kasposi's sarcoma, is a kind of locally aggressive or borderline vascular tumor, whereas ATA is considered as a benign vascular tumor (13).

Hypercalcemia (HC) refers to abnormal serum calcium increment and occurs when the amount of calcium absorbed into extracellular fluid (predominantly through intestine and bone) is much more than that discharged through intestinum 
crassum and kidney (14). Clinical manifestations of HC vary greatly from asymptomatic phenotypes to hypercalcemic crisis, and the latter is life-threatening $(13,15)$. As the calcium balance is the combined effect of regulation through parathyroid hormone $(\mathrm{PTH})$, calcitonin and active vitamin D3 [1,25-(OH)2D3] upon various organs, including intestine, kidney and bones, abnormalities in any segments of this regulatory process may lead to calcium metabolic disturbance (16). Typical causes of HC include hyperparathyroidism (primary, secondary or atopic), vitamin D metabolic disorder, PTH-related protein secretion from tumors and many other pathogenesis, including sarcoidosis, granulomatous, milk alkaline syndrome and adrenal insufficiency (17).

The most common reason of HC syndrome is primary hyperparathyroidism or malignancy-associated HC (18), whereas cases of KMS combined with $\mathrm{HC}$ are extremely rare and have not been reported previously. The present report described the diagnosis and treatment of an infant with KMS combined with HC.

\section{Case report}

Patient data. In September 2015, a 35-day-old male infant with swelling on the upper right arm for $>1$ month and thrombocytopenia for 1 day was admitted to Hunan Provincial People's Hospital (Changsha, China) for the first time. The infant developed a dark purple lump in his upper right arm with a size of $15 \times 4 \times 8 \mathrm{~cm}$ since birth. The guardians (parents of the patient) provided written informed consent for the publication of the clinical information of the patient.

Routine clinical examination. Following hospitalization, a series of routine examinations, including blood routine examination, liver function test, marrow cytology inspection and antiplatelet antibody test, were performed. General lung computed tomography (CT) scans and enhanced CT scanning were also undertaken. In addition, magnetic resonance imaging (MRI) scans of the infant's upper right arm followed by enhanced MRI and magnetic sensitive as well as magnetic resonance venography (MRV) were conducted. Intracranial CT and abdominal ultrasonography were also undertaken when the infant was hospitalized again at 90 days old.

General condition. The patient was born full-term, with normal delivery and precipitate labor. Physical examination indicated that the patient had mild-moderate jaundice of the systemic skin, and dark purple lumps with a length of $\sim 15 \mathrm{~cm}$ were observed surrounding his right arm (Fig. 1). The surface of these lumps was shiny, swollen, and slightly hard and hot to the touch together with few desquamations.

Routine clinical examination results. To understand the patient's condition, routine clinical examination, including physical check, blood routine examination, coagulation function test and liver function examination, were performed. Blood routine examination demonstrated that the hemoglobin level was $81 \mathrm{~g} / 1$ (normal range, 113-151 g/l), hematocrit level was $24.3 \%$ (normal range, 33-45\%), platelet count was $7 \times 10^{9}$ platelets $/ 1$ and mean platelet volume was $8.9 \mathrm{fl}$ (normal range, 6-14 $\mathrm{fl}$ ). The coagulation function test indicated that the fibrin degradation product was $>100 \mu \mathrm{g} / \mathrm{ml}$ (normal range, $0-5 \mu \mathrm{g} / \mathrm{ml}$ ) and D-dimer (DDI) was $>10 \mathrm{mg} / \mathrm{ml}$ (normal range, $0-0.55 \mathrm{mg} / \mathrm{l}$ ), with a prothrombin time of $14.3 \mathrm{sec}$ (normal range, $10.0-15.5 \mathrm{sec}$ ), prothrombin time activity of $85.6 \%$ (normal range, $80-140 \%$ ), prothrombin time-international normalized ratio of 1.246 (normal range, $0.8-1.5$ ), fibrinogen of $0.482 \mathrm{~g} / 1$ (normal range, 2-4 g/l), activated partial thromboplastin time of $43.3 \mathrm{sec}$ (normal range, 24-38.6 sec), thrombin time of $25.4 \mathrm{sec}$ (normal range, 14-21 sec) and antithrombin-III of $63.0 \%$ (normal range, $82-132 \%$ ). Liver function examination demonstrated that alanine aminotransferase and aspartate aminotransferase were normal with total bilirubin, direct bilirubin and indirect bilirubin levels of $145.9,13.2$ and $132.70 \mu \mathrm{mol} / 1$, respectively. Bone marrow cytology results revealed that the patient developed proliferative anemia bone marrow, while laboratory tests presented normal antiplatelet antibodies. General lung CT scans and enhanced CT scanning indicated diffuse lesions in both lungs, therefore, the patient was suspected to have bronchial pneumonia or pulmonary hemorrhage. As illustrated in Fig. 2, the results from MRI scans of the infant's upper arm combined with enhanced MRI, magnetic sensitive and MRV demonstrated occupancy lesions in the right armpit, upper right arm as well as the right elbow, which were potential hemangiomas. The result of blood gas analysis indicated that the $\mathrm{pH}$ value was 7.46, which was slightly higher than the normal range (7.35-7.45).

Treatment course. In line with all the examinations, the infant was diagnosed as KMS with manifestations of giant hemangioma and thrombocytopenia (19). The patient also developed severe anemia with hyperbilirubinemia and disseminated intravascular coagulation. A series of treatments, including heparin anticoagulant, platelet transfusions as well as heparinized cryoprecipitate and fibrinogen supplement were implemented. Methylprednisolone sodium succinate (China National Pharmaceutical Group Corportation, Beijing, China) injection was also conducted, with a dose of $15 \mathrm{mg} / \mathrm{kg}$ per day for 3 days followed by a gradual reduction of dosage to $2 \mathrm{mg} / \mathrm{kg}$ per day for 9 days and sustained for a further 2 months. $\gamma$-globulin antiplatelet antibodies (Nanyue Biological Pharmaceutical Co., Ltd., Hengyang, China) were administered once. Propranolol (Jiangsu Yabang Epson Pharmaceutical Co. Ltd., Changzhou, China) was also prescribed $(0.5 \mathrm{mg} / \mathrm{kg}$ per day and then the amount gradually increased to $2 \mathrm{mg} / \mathrm{kg}$ per day) and stopped before surgery. Subsequently, the patient's condition improved and the patient was discharged with continued oral prednisone (Zhejiang Xianju Pharmaceutical Co. Ltd., Taizhou, China; $2 \mathrm{mg} / \mathrm{kg}$ per day).

The infant was readmitted to our hospital at 90-days-old with repeatedly regurgitating milk associated with cough for 10 days, as well as reduced activity and poor response for 2 days. Physical examination indicated that the infant was $8 \mathrm{KG}$ and presented special features including round face, rubefaction and poor spirit. The infant's right arm was swollen with moderate hardness and normal strength, whereas both his left arm and right leg demonstrated slightly reduced myodynamia. Joints in the overall body were normal without swelling and the pathological meningeal irritation result was 
negative. Head CT scans indicated no abnormalities, nor did the cerebrospinal fluid routine or biochemical examinations.

In order to determine the blood compound contents, serum electrolyte level was detected. The results demonstrated that total calcium was $3.47 \mathrm{mmol} / \mathrm{l}$, which was notably higher than the normal range (2.1-2.9 $\mathrm{mmol} / \mathrm{l})$, whereas sodium was $128.0 \mathrm{mmol} / \mathrm{l}$, which was markedly reduced compared to the normal range (normal range, $137-147 \mathrm{mmol} / \mathrm{l}$ ). The levels of potassium (normal range, $3.5-5.3 \mathrm{mmol} / \mathrm{l}$ ) and chloridion (normal range, 99-110 $\mathrm{mmol} / \mathrm{l}$ ) were 4.85 and $92.1 \mathrm{mmol} / \mathrm{l}$, respectively. Thyroid function was normal and bone alkaline phosphatase was $200 \mathrm{U} / 1$ (normal range, $\leq 200 \mathrm{U} / 1$ ). PTH level was $2.24 \mathrm{pg} / \mathrm{ml}$ while 25 -hydroxy-vitamin D level was normal (20.0 $\mathrm{ng} / \mathrm{ml})$. Urine analysis indicated that the calcium level was $2.78 \mathrm{mmol} / \mathrm{l}$ (normal range: $2.5-7.5 \mathrm{mmol} / \mathrm{l}$ ) whereas the ratio of calcium to creatinine was 1.717 , which was notably higher than the normal range $(<0.21)$. Abdominal ultrasonography demonstrated enhanced renal vertebrae echo with radial arrangement. Treatments including calcium reducing and potassium supplement were performed to correct electrolyte turbulence. Fluid infusion was administered to the infant accompanied with diuretic therapy by furosemide (Shanghai Zhaohui Pharmaceutical Co. Ltd., Shanghai, China) and calcium reducing treatment by alendronate sodium (Hangzhou MSD Pharmaceutical Co. Ltd., Hangzhou, China). Prednisone was taken continuously to increase the potassium level; however, the effect was limited. The blood calcium remained high with fluctuation between 2.8 and $3.37 \mathrm{mmol} / \mathrm{l}$. From all of the above, it was considered that the patient developed cancer-related HC and surgical treatment was advised. Eventually, the hemangioma in the upper right arm of the patient was embolized by microcatheter (Progreat 1.8F, Terumo Corporation, Tokyo, Japan) under general anesthesia on day 22 after the patient was readmitted, according to the manufacturer's protocol. As demonstrated in Fig. 3, the lump markedly reduced in size following surgery. Follow-up at 4 months after surgery indicated that the patient was in good condition.

\section{Discussion}

Infant hemangiomas are the most common benign vascular tumor in infancy (20). Generally, infant hemangiomas may be divided into three phases: Proliferative phase, stabilized phase and involuting phase (21). The majority of hemangiomas are self-limiting, while $10-20 \%$ require additional treatment because of complication generation (22). Several local complications may need to be treated in time as hemangiomas may sometimes develop collapse, necrosis or hemorrhage, while vascular tumor growth in particular parts of the body, such as the upper eyelid, could lead to ametropia, amblyopia or astigmatism (23). Vascular tumors growing near vital organs, including the upper respiratory tract and liver, should be taken into account seriously, since those hemangiomas could cause severe diseases, including acute respiratory failure or congestive heart failure (24). Vascular tumors may also be combined with systemic diseases, and the infant in the present report is a case of hemangiomas associated with thrombocytopenia and coagulopathy.

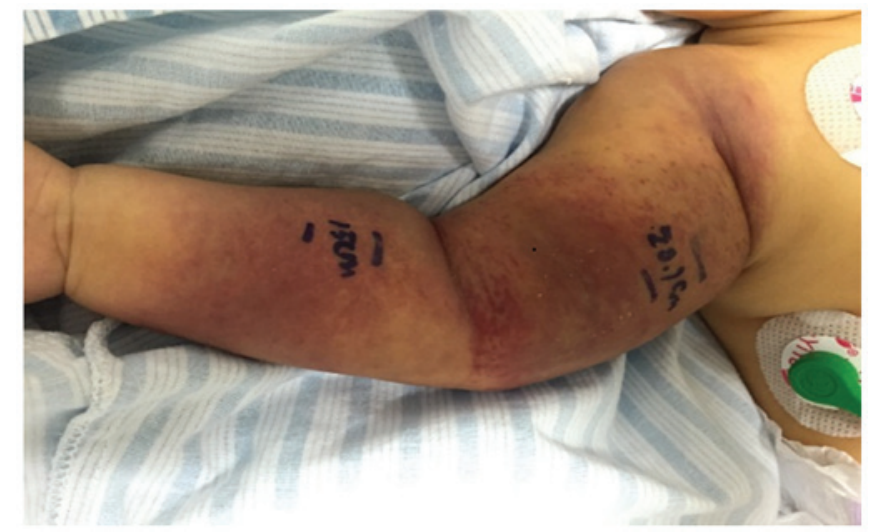

Figure 1. Jaundice in the infant's systemic skin and dark purple lumps surrounding the right arm at the patient's first hospitalization.



B

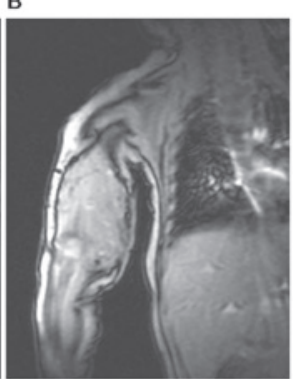

C



Figure 2. MRI plain scanning and enhanced MRI results of the patient at his first hospitalization. By plain scanning, round and sheet-like signal intensity (A) T1 and (B) T2 was identified in the right upper arm and elbow. (C) Enhanced MRI demonstrated that the lesions spread to the armpit, toward spatium intermusculare and subcutaneous fat. The inhomogeneous nature was obvious following enhancement. MRI, magnetic resonance imaging.

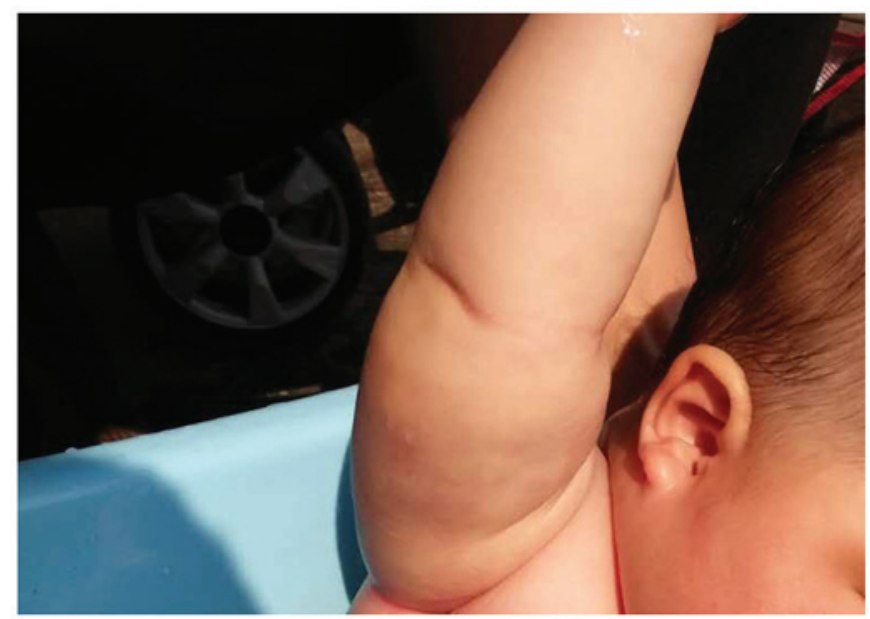

Figure 3. Lumps in the patient's right arm reduced markedly following surgery.

In 1940, Kasabach and Merritt (5) reported a case of a 1-week-old male infant demonstrating widespread purpura and swelling in the left thigh. Biochemical examinations revealed thrombocytopenia and coagulation disorders and results from tissue biopsy diagnosis indicated that the infant had capillary hemangioma. Following this, symptoms 
manifested as a huge capillary hemangioma combined with platelet reduction in the infant, and this was termed KMS (5).

The etiology and pathogenesis of KMS is currently unclear; however, increasing evidence has demonstrated that hemangiomas combined with thrombocytopenia and coagulation disorders are not common vascular tumors (25). The majority of pathological types are KHE and ATA (25-28). ISSVA classified ATA into benign vascular tumors in 2014, while KHE was categorized as locally aggressive or borderline vascular tumors (10).

In the present report, the infant was born with lumps in the upper right arm and the lumps increased suddenly, associated with thrombocytopenia and bleeding tendency. Combined with the confirmation of vascular tumor from radiological technology examination, the infant was diagnosed with KMS. Since KHE and ATA are the most common types of KMS and the use of propranolol during hospitalization had slowed the infant's heart rate to $80 \mathrm{bpm}$, oral prednisone treatment was given to the patient consecutively after his discharge from hospital. Cushing's syndrome-like features were evident in the patient and the hemangioma shrank during the prednisone treatment. At the same time, the patient did not develop thrombocytopenia and coagulation abnormalities.

However, the patient was readmitted again 2 months later due to $\mathrm{HC}$. Multiple calcium reducing treatments were undertaken but the condition did not improve. Results from PTH and vitamin D examination indicated that the patient had neither primary hyperparathyroidism nor vitamin D metabolic disturbance. Since KHE was a kind of borderline tumor, and cases of borderline tumors combined with HC had also been reported previously $(29,30)$, it was speculated whether HC generation was closely related to PTH-related protein (PTHrp) produced by hemangioma. PTHrp is the homologous protein of PTH and shares the same receptor (PTH1 receptor) with PTH to implement similar biological functions $(31,32)$. PTH/PTHrp serves a dual role in bone metabolism and promotes bone formation and resorption, which are predominantly mediated by the protein kinase (PK) A and PKC pathways and dependent on functional regulations of osteoblasts and osteoclasts (33). Although the level of PTHrp was not measured in the present report due to detection limitation, surgical treatment of the hemangioma was recommended for the patient. Due to the large mass of the vascular tumor, resection was not suitable. Therefore, the hemangioma was removed through pipeline arteriosclerosis embolization. Pathological examination was not performed due to the high risk to take pathological specimens. Serum calcium returned to normal range quickly following surgical treatment and the patient was in a good condition at a 4-month follow-up evaluation.

\section{Acknowledgements}

The present study was supported by the Planed Projects of Hunan Provincial Science and Technology Department (grant no. 2013FJ6028) and the Scientific Fund of Health and Family Planning Commission of Hunan Province (grant no. C2013-023).

\section{References}

1. Mulliken JB and Glowacki J: Hemangiomas and vascular malformations in infants and children: A classification based on endothelial characteristics. Plast Reconstr Surg 69: 412-422, 1982.

2. Trenor CC III and Chaudry G: Complex lymphatic anomalies. Semin Pediatr Surg 23: 186-190, 2014.

3. Massarweh S, Munis A, Karabakhtsian R, Romond E and Moss J: Metastatic angiosarcoma and kasabach-merritt syndrome. Rare Tumors 6: 5366, 2014.

4. Croteau SE, Kozakewich HP, Perez-Atayde AR, Fishman SJ, Alomari AI, Chaudry G, Mulliken JB and Trenor CC III: Kaposiform lymphangiomatosis: A distinct aggressive lymphatic anomaly. J Pediatr 164: 383-388, 2014.

5. Kasabach $\mathrm{H}$ and Merritt K: Capillary hemangioma with extensive purpura-Report of a case. Am J Dis Children 59: 1063-1070, 1940.

6. Thomson H: Cutaneous hemangiomas and lymphangiomas. Clin Plast Surg 14: 341-356, 1987.

7. Kim T, Roh MR, Cho S and Chung KY: Kasabach-Merritt syndrome arising from tufted angioma successfully treated with systemic corticosteroid. Ann Dermatol 22: 426-430, 2010.

8. Hall GW: Kasabach-Merritt syndrome: Pathogenesis and management. Br J Haematol 112: 851-862, 2001.

9. Xia H, He Z, Zhu Z, Zhu X, Zhu X, Xie W and Ouyang T: Clinical analysis of 10 cases of Kasabach-Merritt syndrome. Chin J Prac Pediat 26: 125-127, 2011 (In Chinese).

10. Rodriguez V, Lee A, Witman PM and Anderson PA: Kasabach-merritt phenomenon: Case series and retrospective review of the mayo clinic experience. J Pediatr Hematol Oncol 31: 522-526, 2009.

11. Maguiness S and Guenther L: Kasabach-merritt syndrome. J Cutan Med Surg 6: 335-339, 2002.

12. Dasgupta R and Fishman SJ: ISSVA classification. Semin Pediatr Surg 23: 158-161, 2014.

13. Zukerberg LR, Nickoloff BJ and Weiss SW: Kaposiform hemangioendothelioma of infancy and childhood. An aggressive neoplasm associated with Kasabach-Merritt syndrome and lymphangiomatosis. Am J Surg Pathol 17: 321-328, 1993.

14. Thosani S and Hu MI: Denosumab: A new agent in the management of hypercalcemia of malignancy. Future Oncol 11: 2865-2871, 2015.

15. Dellay $B$ and Groth M: Emergency management of malignancy-associated hypercalcemia. Adv Emerg Nurs J 38: $15-25,2016$.

16. Komisarenko IuI: Mineral metabolism and metabolic markers in patients with concomitant endocrine disorders and vitamin D3 deficiency. Lik Sprava: 51-55, 2013 (In Ukrainian).

17. Žofková I: Hypercalcemia. Pathophysiological aspects. Physiol Res 65: 1-10, 2016.

18. Edelson GW and Kleerekoper M: Hypercalcemic crisis. Med Clin North Am 79: 79-92, 1995.

19. Liao Q: Basic and Clinical of Pediatric Hematology. Beijing People's Medical Publishing House, Beijing, pp702-703, 2001.

20. Mulliken JB, Fishman SJ and Burrows PE: Vascular anomalies. Curr Probl Surg 37: 517-584, 2000.

21. Hohenleutner U, Landthaler M, Hamm H and Sebastian G: Hemangiomas of infancy and childhood. J Dtsch Dermatol Ges 5: 334-338, 2007 (In English, German).

22. Cheng CE and Friedlander SF: Infantile hemangiomas, complications and treatments. Semin Cutan Med Surg 35: 108-116, 2016.

23. Ranchod TM, Frieden IJ and Fredrick DR: Corticosteroid treatment of periorbital haemangioma of infancy: A review of the evidence. Br J Ophthalmol 89: 1134-1138, 2005.

24. Masoomi H, Nguyen B, Smith BR, Stamos MJ and Nguyen NT: Predictive factors of acute respiratory failure in esophagectomy for esophageal malignancy. Am Surg 78: 1024-1028, 2012.

25. Kelly M: Kasabach-Merritt phenomenon, Pediatr Clin North Am 57: 1085-1089, 2010.

26. Sarkar M, Mulliken JB, Kozakewich HP, Robertson RL and Burrows PE: Thrombocy topenic coagulopathy (Kasabach-Merritt phenomenon) is associated with Kaposiform hemangioendothelioma and not with common infantile hemangioma. Plast Reconstr Surg 100: 1377-1386, 1997.

27. Enjolras O, Wassef M, Mazoyer E, Frieden IJ, Rieu PN, Drouet L, Taïeb A, Stalder JF and Escande JP: Infants with Kasabach-Merritt syndrome do not have 'true' hemangiomas. J Pediatr 130: 631-640, 1997. 
28. Chiu YE, Drolet BA, Blei F, Carcao M, Fangusaro J, Kelly ME, Krol A, Lofgren S, Mancini AJ, Metry DW, et al: Variable response to propranolol treatment of kaposiform hemangioendothelioma, tufted angioma, and Kasabach-Merritt phenomenon. Pediatr Blood Cancer 59: 934-938, 2012.

29. Boukhris I, Azzabi S, Kéchaou I, Chérif E, Kooli C Romdhane KB, Omar S and Khalfallah N: Hypercalcemia related to PTH-rP revealing malignant hepatic epithelioid hemangioendothelioma. Ann Biol Clin (Paris) 74: 98-102, 2016.

30. Donovan PJ, Achong N, Griffin K, Galligan J, Pretorius CJ and McLeod DS: PTHrP-mediated hypercalcemia: Causes and survival in 138 patients. J Clin Endocrinol Metab 100: 2024-2029, 2015.
31. Okazaki M, Ferrandon S, Vilardaga JP, Bouxsein ML, Potts JT Jr and Gardella TJ: Prolonged signaling at the parathyroid hormone receptor by peptide ligands targeted to a specific receptor conformation. Proc Natl Acad Sci USA 105: 16525-16530, 2008.

32. Wong MH, Stockler MR and Pavlakis N: Bisphosphonates and other bone agents for breast cancer. Cochrane Database Syst Rev: CD003474, 2012

33. Niizuma H, Fujii K, Sato A, Fujiwara I, Takeyama J and Imaizumi M: PTHrP-independent hypercalcemia with increased proinflammatory cytokines and bone resorption in two children with CD-negative precursor B acute lymphoblastic leukemia. Pediatr B1ood Cancer 49: 990-993, 2007. 